SLAC-PUB-9812

May 2003

\title{
Tracking Simulations Near Half-Integer Resonance at PEP-II ${ }^{1}$
}

\author{
Y. Cai and Y. Nosochkov \\ Stanford Linear Accelerator Center, Stanford University, Stanford, CA 94309
}

\begin{abstract}
Beam-beam simulations predict that PEP-II luminosity can be increased by operating the horizontal betatron tune near and above a half-integer resonance. However, effects of the resonance and its synchrotron sidebands significantly enhance betatron and chromatic perturbations which tend to reduce dynamic aperture. In the study, chromatic variation of horizontal tune near the resonance was minimized by optimizing local sextupoles in the Interaction Region. Dynamic aperture was calculated using tracking simulations in LEGO code. Dependence of dynamic aperture on the residual orbit, dispersion and $\beta$ distortion after correction was investigated.
\end{abstract}

Presented at the 2003 Particle Accelerator Conference (PAC 03)

Portland, Oregon, May 12-16, 2003

\footnotetext{
${ }^{1}$ Work supported by Department of Energy contract DE-AC03-76SF00515.
} 


\title{
TRACKING SIMULATIONS NEAR HALF-INTEGER RESONANCE AT PEP-II ${ }^{\dagger}$
}

\author{
Y. Cai, Y. Nosochkov, SLAC, Menlo Park, CA 94025, USA
}

\section{Abstract}

Beam-beam simulations predict that PEP-II luminosity can be increased by operating the horizontal betatron tune near and above a half-integer resonance. However, effects of the resonance and its synchrotron sidebands significantly enhance betatron and chromatic perturbations which tend to reduce dynamic aperture. In the study, chromatic variation of horizontal tune near the resonance was minimized by optimizing local sextupoles in the Interaction Region. Dynamic aperture was calculated using tracking simulations in LEGO code. Dependence of dynamic aperture on the residual orbit, dispersion and $\beta$ distortion after correction was investigated.

\section{INTRODUCTION}

PEP-II [1] has been operating at the betatron tune $\nu_{x} / \nu_{y}$ close to $24.569 / 23.639$ in the High Energy Ring (HER) and 38.649/36.564 in the Low Energy Ring (LER). These working points were selected experimentally for a reliable machine performance, good luminosity and beam lifetime. However, the beam-beam simulations predict that luminosity can be increased by operating betatron tune very close and above the half-integer resonance. Fig. [1]shows the LER tune diagram with synchro-betatron resonances up to the 4 th order and a contour plot of the single bunch luminosity. Calculation of luminosity was done using the beam-beam code developed at SLAC [2] which has been recently upgraded to the three dimensional version.

The difficulty of operating close to half-integer resonance comes from enhancement of the resonance effects on betatron motion. It is well known that perturbation of $\beta$ function created by focusing errors depends on tune $\nu$ as

$$
\frac{\Delta \beta}{\beta}(s)=\frac{1}{2 \sin 2 \pi \nu} \oint \beta(l) \Delta K_{1}(l) \cos 2 \phi(s, l) d l,
$$

where $\mu$ is phase advance, $\phi(s, l)=\pi \nu-|\mu(s)-\mu(l)|$, and $\Delta K_{1}$ is a focusing error created mainly by quadrupole field imperfections, horizontal orbit at sextupoles, and momentum error. Close to half-integer resonance, growth of $\Delta \beta / \beta$ comes from the resonance term $[\sin 2 \pi \nu]^{-1}$ which behaves as $1 / \Delta \nu$ when distance to the resonance is as small as $\Delta \nu \ll 1 / 2 \pi$. On the other hand, orbit and dispersion are not excited by the half-integer resonance.

For significant enhancement of luminosity, fractional value of horizontal tune should be in the range of $\left[\nu_{x}\right] \approx .51$. At this working point, enhancement of $\Delta \beta_{x} / \beta_{x}$ in HER and LER due to the resonance term in Eqn. 1 would be a

\footnotetext{
${ }^{\dagger}$ Work supported by Department of Energy contract DE-AC03$76 \mathrm{SF} 00515$
}

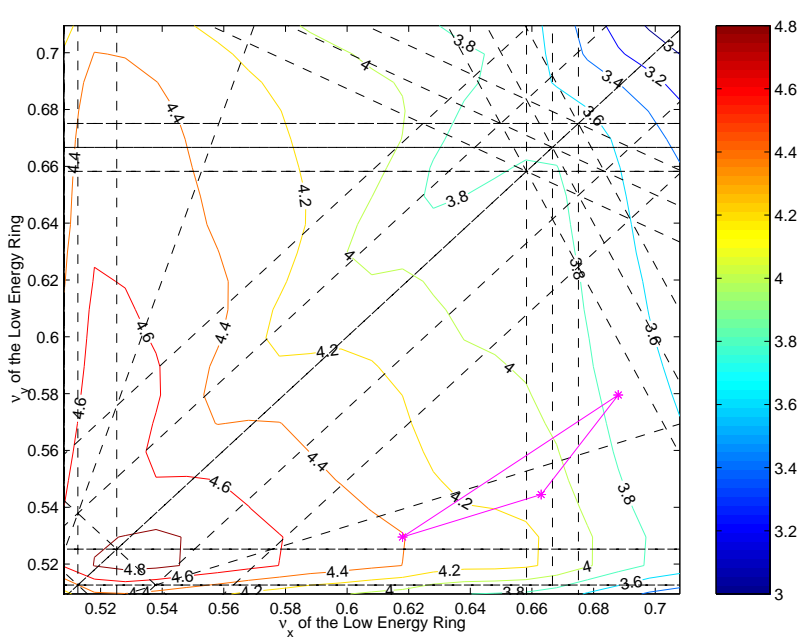

Figure 1: Single bunch luminosity scan $\left[10^{30} \mathrm{~cm}^{-2} \mathrm{~s}^{-1}\right]$.

factor of 6.7 and 12.8 , respectively, compared to the present tune. Without compensation, the large $\beta$ growth may significantly increase amplitude dependent non-linear aberrations and reduce dynamic aperture and beam lifetime.

More resonance effects are generated by the synchrotron sidebands of the half-integer resonance: $2 \nu_{x}+m \nu_{s}=n$, where $\nu_{s}$ is a synchrotron tune, and $m, n$ are integers. In the LER, where $\nu_{s}=0.025$, the 1st and 2nd synchro-betatron resonances occur at $\left[\nu_{x}\right]=.5125$ and .525 , while in HER with $\nu_{s}=0.045$ the 1 st sideband is at $\left[\nu_{x}\right]=.5225$. Tracking simulations will show that the sidebands have a strong effect on dynamic aperture, therefore working tune should be chosen reasonably far from them. In addition, variation of tune with synchrotron momentum oscillations should be minimized to avoid crossing with these resonances.

Optimization of PEP-II lattice near half-integer resonance and analysis of dynamic aperture are discussed below. The optics with $\beta_{x}^{*} / \beta_{y}^{*}=50 / 1.25 \mathrm{~cm}$ at the Interaction Point (IP) is used.

\section{LATTICE OPTIMIZATION}

PEP-II has two tuning sections which can be locally adjusted to change betatron tune without affecting the rest of machine optics. Initially, only these sections were modified to move the horizontal tune closer to half-integer, and vertical tune to $\left[\nu_{y}\right] \approx .61$ as suggested by beam-beam analysis. But tracking simulations showed that dynamic aperture was not sufficiently large with machine errors and synchrotron momentum oscillations of up to $\pm 8 \sigma_{p}$, where $\sigma_{p}$ is the rms value of relative momentum spread $\frac{\Delta p}{p}$ in the beam. Analysis of chromaticity indicated that non-linear variation of horizontal tune with momentum needs to be 


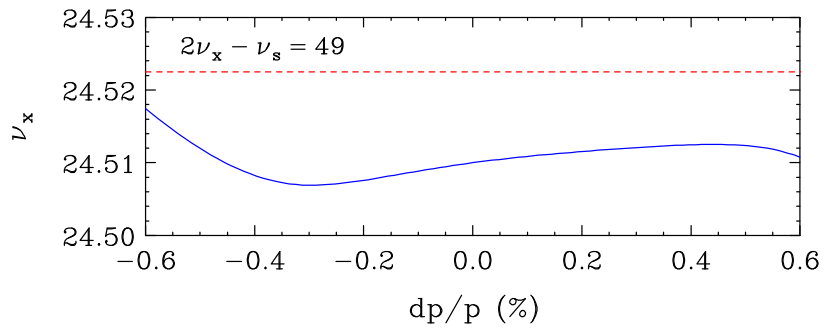

Figure 2: HER horizontal tune vs. $\frac{\Delta p}{p}$ at $\nu_{x}=24.51$.

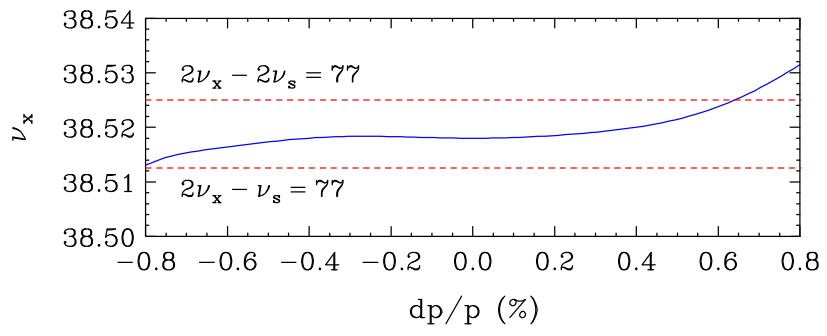

Figure 3: LER horizontal tune vs. $\frac{\Delta p}{p}$ at $\nu_{x}=38.518$.

further reduced to avoid crossing with the synchro-betatron resonances.

In PEP-II, the most contribution to non-linear chromaticity is generated in the final quadrupole doublets near IP. This chromaticity is compensated by the Interaction Region (IR) sextupoles located in the same phase with the doublets. Variation of strength of these sextupoles allows to compensate quadratic dependence of tune on $\frac{\Delta p}{p}$, and a small adjustment of sextupole phase advance helps reduce the higher order variation.

Minimum of the second order chromaticity was achieved by reducing strengths of the IR sextupoles correcting horizontal chromaticity. Further improvement in LER resulted from reduction of horizontal phase advance between the IR horizontal sextupoles and IP by $5^{\circ}$. For correction of the machine linear chromaticity, strength of the global sextupoles was increased to compensate for the weaker IR sextupoles. Because the adjusted IR sextupoles in LER have a non-zero design orbit, the reduced sextupole strength created a feed-down effect of linear focusing and coupling. This small perturbation was compensated by a slight adjustment of the IR magnet strengths.

The optimized horizontal tune for momentum range of $-10 \sigma_{p}<\frac{\Delta p}{p}<10 \sigma_{p}$ is shown in Fig. 2] 3. where the working point is $\nu_{x} / \nu_{y}=24.51 / 23.61$ in HER and $38.518 / 36.61$ in LER. The straight dash lines depict the half-integer synchrotron sidebands. In HER, a positive linear chromaticity $\xi=+1$ was used in Fig. 2 to counteract the negative slope of non-linear tune variation. Due to the large synchrotron tune, it was possible to place the HER working point below the 1st synchrotron sideband without crossing with the resonance lines. In the LER, synchrotron tune is a factor of 2 smaller while energy spread is $25 \%$ larger, therefore the closest to half-integer working point was chosen between the 1 st and 2 nd sidebands.

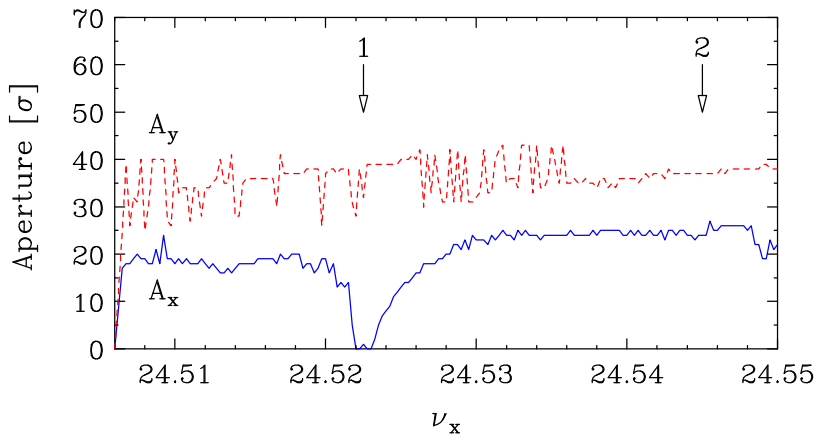

Figure 4: HER dynamic aperture without magnet errors vs. $\nu_{x}$ at $\nu_{y}=23.61$. Synchrotron sidebands: 1) $2 \nu_{x}-\nu_{s}=49$, 2) $2 \nu_{x}-2 \nu_{s}=49$.

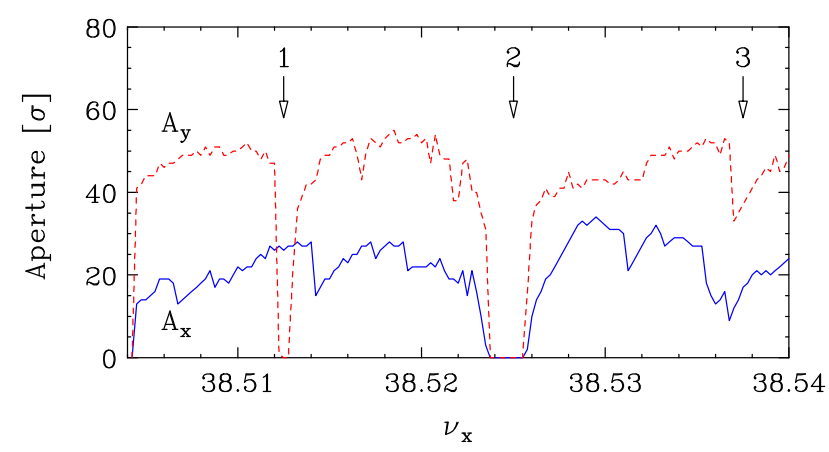

Figure 5: LER dynamic aperture without magnet errors vs. $\nu_{x}$ at $\nu_{y}=36.61$. Synchrotron sidebands: 1) $2 \nu_{x}-\nu_{s}=77$, 2) $\left.2 \nu_{x}-2 \nu_{s}=77,3\right) 2 \nu_{x}-3 \nu_{s}=77$.

\section{DYNAMIC APERTURE SIMULATIONS}

Calculations of dynamic aperture were performed using tracking simulations in LEGO code [3]. First, dependence of aperture on betatron tune near half-integer resonance was investigated for lattice without magnet errors, but with synchrotron momentum oscillations of $\pm 8 \sigma_{p}$. The resultant horizontal tune scan is shown in Fig. 4 5 for HER and LER, where dynamic aperture is normalized by the $r m s$ size of a fully coupled beam.

The HER horizontal dynamic aperture vanishes in the vicinity of the main half-integer resonance $2 \nu_{x}=49$ and its 1 st sideband. In LER, the strongly affecting resonances are $2 \nu_{x}+m \nu_{s}=77$ with $m=0,-1,-2,-3$. Naturally, the working tune should be chosen reasonably far from these resonances. The following scenarios for more and less aggressive horizontal tune $\nu_{x}$ near half-integer were investigated in the simulations:

1. HER: 24.51, LER: 38.518.

2. HER: 24.529, LER: 38.529 .

Dynamic aperture scan versus vertical tune was performed for the first scenario of $\nu_{x}$ and the range of $\left[\nu_{y}\right]$ from .55 to .64 . It showed that dynamic aperture gradually reduces as $\left[\nu_{y}\right]$ becomes closer to $\left[\nu_{x}\right]$ and the working point approaches the crossing of half-integer and coupling resonances. Based on this scan, the vertical fractional tune of $\left[\nu_{y}\right]=.61$ was chosen for these simulations. A lower $\nu_{y}$ may be considered for further luminosity enhancement. 
Secondly, tracking simulations with field errors, misalignment and $\frac{\Delta p}{p}= \pm 8 \sigma_{p}$ synchrotron momentum oscillations were performed for the selected working points. For statistics, ten different settings ("seeds") of random machine errors were used in each tracking. Perturbation of beam orbit, linear chromaticity, betatron tune and vertical dispersion was compensated using realistic correction schemes in LEGO. Since distortion of $\beta$ function becomes more sensitive to focusing errors near half-integer resonance, a special correction of $\frac{\Delta \beta}{\beta}$ was implemented in LEGO. It uses MICADO method to find the most effective quadrupoles to minimize $\beta$ perturbation.

Due to the greater effect of errors near half-integer resonance, a better machine correction is needed to maintain acceptable dynamic aperture. To verify tolerance to various errors, simulations were performed for different levels of machine correction. It has been confirmed that beam orbit should be decreased for an acceptable dynamic aperture. The better orbit correction reduces the feed-down focusing errors in sextupoles as well as residual dispersion in the machine. On the other hand, correction of vertical dispersion did not significantly affect dynamic aperture in the observed range of residual $\mathrm{rms} \Delta \eta_{y}$ from $\sim 70$ to $5 \mathrm{~mm}$.

As expected, the simulations confirmed that compensation of $\Delta \beta / \beta$ is required in the first scenario, where $\nu_{x}$ is closer to the resonance. Typically, $\Delta \beta / \beta$ was corrected to the $r m s$ level of $<5 \%$. In the second scenario, at $\left[\nu_{x}\right]=.529$, correction of $\beta$ function was less important, although it helped to improve cases with small aperture. Table 1 summarizes the observed approximate levels of $\mathrm{rms}$ orbit and $\Delta \beta_{x} / \beta_{x}$ for acceptable dynamic aperture.

Table 1: Tolerances on $r m s$ orbit and $\Delta \beta_{x} / \beta_{x}$.

\begin{tabular}{|c|c|c|c|c|}
\hline & \multicolumn{2}{|c|}{ HER } & \multicolumn{2}{c|}{ LER } \\
\hline$\left[\boldsymbol{\nu}_{\boldsymbol{x}}\right]$ & $\mathbf{. 5 2 9}$ & $\mathbf{. 5 1 0}$ & $\mathbf{. 5 2 9}$ & $\mathbf{. 5 1 8}$ \\
\hline orbit $(\mathrm{mm})$ & 1 & 1 & 0.4 & 0.2 \\
$\Delta \beta_{x} / \beta_{x}(\%)$ & 25 & 5 & 25 & 5 \\
\hline
\end{tabular}

The resultant dynamic aperture in HER at $\nu_{x} / \nu_{y}=$ $24.51 / 23.61$ for a good level of correction is shown in Fig. 6 at the injection point. Stable particle motion corresponds to the area inside the dash lines which represent 10 different seeds of random machine errors. The solid halfellipse, shown for reference, is the $10 \sigma$ size of a fully coupled beam at injection with emittance $\epsilon_{x}=48 \mathrm{~nm}$ and $\epsilon_{y}=$ $\epsilon_{x} / 2$. In this simulation, the residual rms orbit, dispersion and $\beta$ distortions after correction were: $0.27 / 0.31 \mathrm{~mm}$, $\Delta \eta=31 / 8 \mathrm{~mm}$ and $\Delta \beta / \beta=1.9 / 1.5 \%$ in $x / y$ planes, respectively. Linear chromaticity was set to +1 to minimize non-linear tune variation with momentum.

The LER dynamic aperture at $\nu_{x} / \nu_{y}=38.518 / 36.61$ is shown in Fig. [7 at the injection point. The solid halfellipse corresponds to $10 \sigma$ size of a fully coupled beam at injection with emittance $\epsilon_{x}=24 \mathrm{~nm}$. The residual rms orbit, dispersion and $\beta$ distortions after correction were: $0.20 / 0.23 \mathrm{~mm}, \Delta \eta=23 / 9 \mathrm{~mm}$ and $\Delta \beta / \beta=2.4 / 2.5 \%$ in $x / y$ planes, respectively. Linear chromaticity was set to

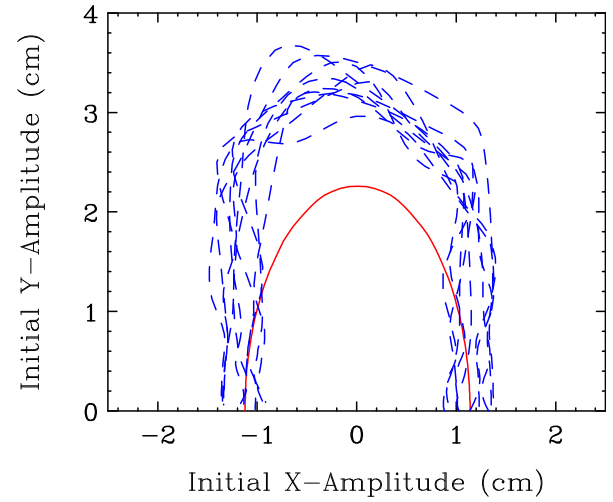

Figure 6: HER dynamic aperture at $\nu_{x} / \nu_{y}=24.51 / 23.61$.

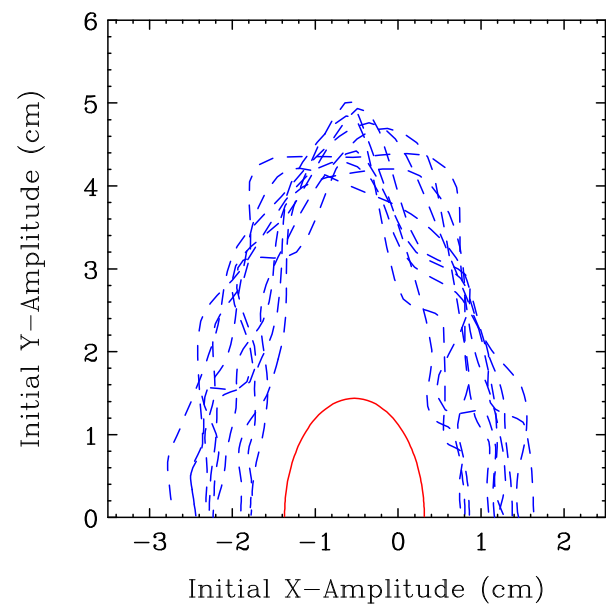

Figure 7: LER dynamic aperture at $\nu_{x} / \nu_{y}=38.518 / 36.61$.

zero in this case.

Implementation of the tune near half-integer resonance has been recently performed at PEP-II. The working point was successfully moved to $\nu_{x} / \nu_{y}=24.52 / 23.63$ in HER and 38.52/36.57 in LER. After the necessary machine adjustments luminosity has been improved by $\sim 15 \%$ to the new record of $6.1 \cdot 10^{33} \mathrm{~cm}^{-2} \mathrm{~s}^{-1}$.

\section{CONCLUSION}

Beam-beam simulations performed for PEP-II predicted an enhancement of luminosity for a betatron tune near a half-integer resonance. Horizontal fractional tune of .52 has been recently implemented at PEP-II and $\sim 15 \%$ luminosity gain has been achieved. Particle tracking simulations showed that an improved machine correction is needed for acceptable dynamic aperture at $\left[\nu_{x}\right]=.51$ in HER and .518 in LER. This requires a minimization of non-linear chromaticity and tighter correction of orbit and $\beta_{x}$ distortions. At $\left[\nu_{x}\right]=.529$, a looser orbit correction may be used while compensation of $\Delta \beta_{x}$ may not be necessary.

\section{REFERENCES}

[1] PEP-II Conceptual Design Report, SLAC-418, 1993.

[2] Y. Cai, et al., Phys. Rev. ST Accel. Beams 4, 011001 (2001).

[3] Y. Cai, et al., SLAC-PUB-7642, 1997. 